\title{
Palliative Intervention for Mitigating Fatigue and Sleep Inefficiency among Chronic Obstructive Pulmonary Disease Clients
}

\author{
Amany Mohamed Saad ${ }^{1, *}$, Enas Ebrahiem E. Abo Elfetoh ${ }^{2}$ \\ ${ }^{1}$ Community Health Nursing Department, Faculty of Nursing, Helwan University, Egypt \\ ${ }^{2}$ Adult Health Nursing Department, Faculty of Nursing, Helwan University, Egypt \\ *Corresponding author: amany1234564@yahoo.com \\ Received March 21, 2019; Revised May 04, 2019; Accepted May 24, 2019
}

\begin{abstract}
Chronic obstructive pulmonary disease is a public health problem cause mortality. Palliative intervention is supportive in managing, and helping clients living with chronic obstructive pulmonary disease. Aim: This study aims to evaluate the effect of palliative care intervention for mitigating fatigue and sleep inefficiency among chronic obstructive pulmonary disease clients. Design: A quasi-experimental design was used. Setting: The study was conducted in Chest Outpatient Clinic, Ain Shams University Hospitals. Sample: A purposive sample comprised of 70 clients. Tools: A structured interview questionnaire was used to collect the data and included three parts: Part I. Client's demographic characteristics. Part II. The Pittsburgh Sleep Quality Index. Part III. Fatigue Severity Scale. Results: The sleep quality index was improved from $7.1 \%$ of clients had good sleep quality pre intervention to $85.7 \%$ post intervention and also fatigue severity was improved from $90 \%$ of them had high fatigue pre intervention to $4.3 \%$ post intervention with highly statistically significant positive correlation between total fatigue and sleep quality index among chronic obstructive pulmonary disease clients $(\mathrm{p}=<0.001)$. Conclusion: The palliative care intervention had a positive effect on chronic obstructive pulmonary disease clients and helped them to mitigate sleep inefficiency, which led to reduce fatigue. Recommendations: An illustrated booklet containing information about benefits of gradual relaxation exercises should be available in all chest outpatient clinics. Training of the nurses on how to apply the palliative care intervention to chronic obstructive pulmonary disease clients.
\end{abstract}

Keywords: palliative, mitigating fatigue, sleep inefficiency, chronic obstructive pulmonary disease

Cite This Article: Amany Mohamed Saad, and Enas Ebrahiem E. Abo Elfetoh, "Palliative Intervention for Mitigating Fatigue and Sleep Inefficiency among Chronic Obstructive Pulmonary Disease Clients." American Journal of Nursing Research, vol. 7, no. 4 (2019): 509-518. doi: 10.12691/ajnr-7-4-13.

\section{Introduction}

Chronic obstructive pulmonary disease (COPD) is a common public health problem and considered an essential cause of morbidity and mortality in both developed and developing countries. The COPD is expected to be the third leading cause of death by 2030 [1]. Chronic obstructive pulmonary disease (COPD) is the most common serious lung disease in the United States(US) over the last few decades. There are 12 to 16 million adults in the United States diagnosed with COPD, it is about $6.4 \%$ of the US population and an equal number of Americans with COPD may still be undiagnosed. As well, 3 million people worldwide die from COPD annually $[2,3]$.

Its prevalence, morbidity, and mortality vary across countries and different groups within countries [4].

Chronic obstructive pulmonary disease is a condition that makes it difficult to move air into and out of a person's lungs, this is called airflow obstruction or resistance. The COPD is characterized by a progressively increasing airflow obstruction that cannot be fully reversed, although it can sometimes be temporarily improved by medications. In almost all cases, COPD has been caused by the long-term inhalation of pollutants, especially cigarette smoke. This obstruction in airflow of individuals with COPD experience symptoms such as dyspnea, coughing, sleep disturbances, and fatigue $[5,6]$.

Fatigue is a common symptom in COPD. It is a subjective and multidimensional symptom that incorporates total body feelings ranging from tiredness to exhaustion, this condition interferes with individuals' ability to do their body functions [7]. Chronic obstructive pulmonary disease is one of the chronic systemic diseases that affects on people's sleep routine and quality [8]. Sleep is one of basic needs of human and has important role in individual's health and productive life [9].

Poor sleep quality and quantity could be attributed to stressful conditions and release of epinephrine and norepinephrine, resulting in increased heart rate, blood pressure, and respiratory rate [10]. Breathing exercises in clients with COPD, such as pursed-lip breathing and 
diaphragmatic breathing, aim to improve airway function and respiratory pattern through reducing respiratory rates [11].

Palliative care intervention is a multidiscipline specialty that is used to improve quality of life for both clients and their families, focused on clients with a serious disease and helping to mitigate the symptoms and stress of their disease, and can be provided along with curative treatment [12].

Palliative care intervention becomes a valuable assistant of therapeutic care for clients with COPD in the absence of curative treatment, progressive dyspnea, and the use of opioids for the treatment of severe and difficult symptoms. This is a prime motivator of therapy. Clients with COPD experience psychological and emotional suffering and high morbidity and mortality rates. In spite of this, they don't always receive adequate palliative care due to lack of knowledge about it [13].

As well palliative care intervention, known as supportive care provided across outpatient settings that focus on mitigate suffering and providing comfort, it is basic in managing chronic obstructive pulmonary disease. This care is focused on helping to achieve the best possible quality of life. It is appropriate for all clients living with COPD regardless of age, stage or prognosis [14].

Nurses have a huge role in promoting healthy behavior for clients with COPD by providing advice, educating, and supporting them and their families, and encouraging management of their disease. The nurse also assists the individual to use controlled breathing techniques, including pursed-lip breathing, the forward leaning position, and abdominal breathing, to control dyspnea and anxiety. The goal is a reduced respiratory rate, and enhanced expiratory tidal volume, thus decreasing air trapping [15]. Nurses who work in palliative care intervention are specially trained to deal with complex pain and symptoms, as well as communication about serious illness, improve understanding of treatment options and match their goals to those options, also help support family caregivers and provide practical support [16].

\subsection{Significance of the Study}

Chronic obstructive pulmonary disease is one of the main causes of global mortality. It is estimated that 210 million clients are living with this disease, and COPD is predicted to become the third leading cause of death in the world by 2030 [17]. The prevalence of chronic obstructive pulmonary disease in the Middle East and North Africa is around $4 \%$ in adults. As well, 3 million Egyptians are at risk of chronic obstructive pulmonary disease, accounting for about $3.9 \%$ of population. However, prevalence and mortality information is not available [18].

Today, a supportive non-pharmacological method as relaxation and breathing exercises for obstructive pulmonary disease focusing on the clients' needs to help them achieve independence and maintain the maximum level of autonomy and function in society [18]. So palliative care intervention is important for mitigating fatigue and sleep inefficiency among chronic obstructive pulmonary disease clients.

\section{Aim of the Study}

The study aimed to evaluate the effect of palliative care intervention for mitigating fatigue and sleep inefficiency among chronic obstructive pulmonary disease (COPD) clients.

\subsection{Hypotheses}

H1. Palliative care intervention will mitigate fatigue severity and sleep inefficiency to COPD clients.

H2. There will be a significant relation between fatigue severity and sleep quality to COPD clients.

\subsection{Subjects and Methods}

\subsubsection{Design}

A quasi experimental study design was utilized.

\subsubsection{Setting}

The study sample was recruited from the Chest Outpatient Clinic, affiliated to Ain Shams University Hospitals, Egypt.

\subsubsection{Sample}

A purposive sample comprised of 70 clients, who were selected according to the following inclusion criteria:

- Conscious adult, age from 20-40 years .

- Willing to collaborate and participate in the study.

- Diagnosed with COPD since 6 months or more.

- Client with no co-morbid conditions.

- Ability to read and write.

\section{Sample Size Calculation:}

The sample size estimated by using the following equation:

$$
n=\frac{N \times p(1-p)}{\left[\left[N-1 \times\left(d^{2} \div z^{2}\right)\right]+p(1-p)\right]}
$$

$\operatorname{Nxp}(1-\mathrm{p})=(761 *(0.053 *(1-0.053))) /$

$\mathrm{N}-1=(761-1)^{*}$

$\mathrm{d}^{2} / \mathrm{z}^{2}=0.0025 / 3.8416$

$\mathrm{p}(1-\mathrm{p})=0.053 *(1-0.053)$

$\mathrm{n}=70$

Where:

$\mathrm{n}=$ Sample size .

$\mathrm{N}=$ Community size (clients attended chest outpatient clinic in the year 2017).

$\mathrm{z}=$ Class standard corresponding to the level of significance.

$\mathrm{d}=$ The margin error rate.

$\mathrm{p}=$ Ratio provides a neutral property $=0.053$ [20].

Based on the equation, the sample size is 70 clients who participated in the study.

\subsubsection{Tools for Date Collection}

A structured interview questionnaire was used in pre and post intervention. It was constructed by the researchers in simple Arabic language, and consisted of three parts. 
Part I: Client's demographic characteristics such as: Age, gender, educational level and family income, marital and working status, duration of disease... etc.

Part II: The Pittsburgh Sleep Quality Index (PSQI): It was adapted from [21] to measure the quality and patterns of sleep for the clients.

\subsubsection{Scoring System}

The Pittsburgh Sleep Quality index (PSQI) contains 19 questions combined to form seven components (sleep quality, latency, duration, efficiency, disturbances, sleep with medication, and daytime dysfunction). The client's answers point to the most accurate answer for the majority of days and nights in the past month. Each of which has a range of 0-3 points. The seven component scores are then added to yield the total score, with a range of 0-21 points, zero indicating no difficulty and twenty one indicating sever difficulties. The total scores were categorized as the following:

Poor PSQI $>75 \%$ with score ranged from $\geq 17-21$

Average PSQI 50-75\% with score ranged from 11-16

Good PSQI $<50 \%$ with score ranged from 0-10

NB: Sleep efficiency $=($ hours slept/hours in bed $) \times 100 \%$

Part III: Fatigue Severity Scale (FSS): The FSS was adopted from [22]. It contains nine items that explore severity of fatigue symptoms.

\subsubsection{Scoring System}

The items were scored in five-point Likert scale. The scale ranging from strongly disagree takes one, disagree takes two, neutral takes three, agree takes four, strongly agree takes five points. The client read each statement and circle a number from 1 to 5 , depending on how he/she felt during the past week. A low value indicates disagreement, while a high value indicates agreement. The total scores were categorized as the following:

Low fatigue severity $<50 \%$ with score ranged from $\leq 22$

Average fatigue severity $50-75 \%$ with score ranged from 23-34

High fatigue severity $>75 \%$ with score ranged from $\geq 35-45$

\subsection{Content Validity}

Testing validity of the tools was reviewed by a panel of five experts in the Community Health Nursing and Medical Surgical Nursing fields to ascertain their face, clarity, completeness and relevance of the content.

\subsection{Reliability of the Tools}

Testing reliability of proposed tools was done statistically as the following: The PSQI tool Cronbach's alpha was 0.83, and FSS: Cronbach's Alpha was 0.88 , this indicate high reliability of the tools.

\subsection{Pilot Study}

A pilot study was carried out on $10 \%$ (7) of the total number of the study sample to test the applicability, clarity and efficacy of the tools, also to assess the time required for filling in the tools before beginning the actual data collection. According to the results of the pilot study, no modifications were done on the tools of the study. So, those clients who participated in the pilot study were included in the main study sample.

\subsection{Ethical Considerations}

The purpose of the study was explained to the hospital director in order to get approval to apply this study for clients in the Chest Outpatient Clinic. As well, the researchers explained the aim of the study to each client and got consent regarding participation in the study. Clients who agreed to participate were assured that the obtained information will be treated confidentially and used only for the purpose of the study. They were also informed that they have the right to refuse or withdraw at any time from the study without giving any reason.

\subsection{Field Work}

The official approvals were obtained from the administrators of Ain Shams University Hospitals for conducting the study in the Chest Outpatient Clinic. Letters of request were issued to them from the Faculty of Nursing, Helwan University, containing the aim of the study and its expected outcomes. Each client involved in the study was interviewed individually, the purpose of the study was explained to them. The intervention was developed by the researchers based on reviewing the recent and related literature and tool assessment (pre-test).

The study data collection took a period of nine months from the beginning of December 2017 to the end of August 2018, about 20-25 minutes were necessary to complete the tools. The researchers were available in the Chest Outpatient Clinic three days/week, from 9.00 a.m. to 1.00 p.m.

The application of palliative care intervention was conducted through four phases: Assessment, planning, implementation, and evaluation phase.

\section{1- Assessment phase}

The beginning phase was done by using the pre-test after the approval taken to carry out the study, the researchers greeted the clients, introduced themselves for them also, they explained the aim of the study. An analysis of the obtained pretest data was then done to help with the design of the palliative care intervention. In the pre-test, data were collected by applying the demographic data, PSQI and FSS for clients who were attending to the Chest Outpatient Clinic.

\section{2- Planning phase:}

The intervention was designed according to general objective to mitigate fatigue and sleep inefficiency for clients with COPD, this achieved through application of palliative care intervention by performing deep breathing and relaxation exercises, this based on analysis of the actual clients' needs in pre assessment by using the pre-test tools.

A booklet was prepared by the researchers, in simple Arabic language and consistent with the related literatures. As well, the intervention met clients' needs and their understanding level. This booklet included: Meaning, benefits of exercises for COPD clients, purpose of deep breathing, positive effects, and the practices that take into consideration during deep breathing exercises, information 
about how to perform gradual relaxation, respiration control, and relaxation exercises.

\section{3- Implementation phase:}

The palliative care intervention was presented in sessions. The total number of clients was 70 divided into 10 groups, each group comprised 7 clients, the intervention was applied in 4 sessions for each group (2 theoretical \& 2 practical). Each session takes from 35-45 minutes according to the items presented.

The educational $\mathrm{CD}$ on palliative care intervention include: Correct deep breathing and relaxation exercises technique, developed by the researchers, was given to clients. The researchers met the clients with COPD in a quiet and special room in the Chest Outpatient Clinic and then allowed them to listen and watch a CD and the researchers did these exercises, and then the clients were asked to do them. Each client was given the booklet and $\mathrm{CD}$ and asked to do these exercises in the home once a day for hours till they felt improved. After two months the client were asked in telephone to come to outpatient clinic to assessed in the post test. The methods of teaching included a lecture, followed by group discussions in addition to listen and watch a CD, and practices. Media of teaching were the booklet and CD.

\section{4- Evaluation phase:}

Effect of the palliative care intervention on the studied clients was evaluated as follows: The quality and efficiency of sleep and fatigue severity for the client were measured by using post-test, immediately after finishing all sessions of the intervention for one time only by using the same tool.

\subsection{Statistical Analysis}

Recorded data were analyzed using the Statistical Package for Social Sciences (SPSS), version 20.0. Quantitative data were expressed as mean and standard deviation (SD). Qualitative data were expressed as frequency and percentage. Paired sample t-test was used when comparing between related sample. Chi-square $\left(\mathrm{X}^{2}\right)$ test was used to compare proportions between two qualitative parameters. Spearman's rank correlation coefficient (r) was used to assess the degree of association between two sets of variables. P-value $\leq 0.05$ was considered significant, and $\mathrm{P} \leq 0.001$ was considered as highly significant.

\subsection{Results}

Table 1: This table reveals that $31.4 \%$ of the clients were aged $20-30$ years, while $68.6 \%$ of them were $>30-40$ years. Regarding gender, $62.9 \%$ of the clients were male. Concerning educational level, an equal percentage of $40.0 \%$ of them were either primary, or university, and $71.4 \%$ were married. As for family level of income, $77.1 \%$ were low income and $22.9 \%$ were moderate income. concerning working status, $57.1 \%$ of them were employed, and related to working type more than half of them were working as carpenters, bakers, and shoe repair. As regards duration of disease, $48.5 \%$ of them had the disease from $1-5$ years, and $22.9 \%$ had it from $>10$ years. Regarding to smoking, $40.0 \%$ were smoker, and $71.4 \%$ of the smokers intended to quit smoking.
Table 1. Distribution of Client's with Chronic Obstructive Pulmonary Disease According to their Demographic Characteristics $(\mathbf{n}=\mathbf{7 0})$

\begin{tabular}{|c|c|c|}
\hline Demographic characteristics & No & $\%$ \\
\hline Age (years) & & \\
\hline $20-30$ & 22 & 31.4 \\
\hline$>30-40$ & 48 & 68.6 \\
\hline Mean \pm SD & \multicolumn{2}{|c|}{$32.86 \pm 6.90$} \\
\hline Gender & & \\
\hline Male & 44 & 62.9 \\
\hline Female & 26 & 37.1 \\
\hline Educational level & & \\
\hline Primary education & 28 & 40.0 \\
\hline Preparatory and secondary education & 14 & 20.0 \\
\hline University & 28 & 40.0 \\
\hline Marital status & & \\
\hline Single & 16 & 22.9 \\
\hline Married & 50 & 71.4 \\
\hline Widowed & 4 & 5.7 \\
\hline Family level of income & & \\
\hline Low income than expenditure & 54 & 77.1 \\
\hline Moderate income and expenditure & 16 & 22.9 \\
\hline High income than expenditure & 0 & 0.0 \\
\hline Working status & & \\
\hline Employed & 40 & 57.1 \\
\hline Unemployed & 30 & 42.9 \\
\hline Working type $(n=40)$ & & \\
\hline Carpenters & 17 & 42.5 \\
\hline Bakers & 10 & 25.0 \\
\hline Shoe repair & 13 & 32.5 \\
\hline Duration of disease (in years) & & \\
\hline $1-5$ & 34 & 48.5 \\
\hline $6-10$ & 20 & 28.6 \\
\hline$>10$ & 16 & 22.9 \\
\hline Family history of disease & & \\
\hline Yes & 24 & 34.3 \\
\hline No & 46 & 65.7 \\
\hline Smoking & & \\
\hline Smoker & 28 & 40.0 \\
\hline Non-smoker & 42 & 60.0 \\
\hline Quit of smoking (n=28) & & \\
\hline Yes & 20 & 71.4 \\
\hline No & 8 & 28.6 \\
\hline
\end{tabular}

Table 2: Shows that there were highly statistically significant differences in all components of PSQI scores pre/post palliative care intervention application $(\mathrm{p}=<0.001)$.

Figure 1: Indicates that $7.1 \%$ of the clients had good sleep quality index pre intervention and improved to $85.7 \%$ of them having good sleep quality post intervention with a highly statistically significant difference in comparison to pre palliative intervention $(\mathrm{P}=<0.001)$.

Table 3: Demonstrates that there were highly statistically significant differences in all items of fatigue severity scores pre/post palliative care intervention application $(\mathrm{p}=<0.001)$. 
Table 2. Descriptive Statistics for Comparison between PSQI Scores Pre and Post Palliative Care Intervention (n=70)

\begin{tabular}{|c|c|c|c|c|c|c|c|}
\hline \multirow{2}{*}{\multicolumn{2}{|c|}{ PSQI components }} & \multicolumn{2}{|c|}{ Pre } & \multicolumn{2}{|c|}{ Post } & \multicolumn{2}{|c|}{ Chi-square } \\
\hline & & No & $\%$ & No & $\%$ & $\mathbf{X}^{2}$ & $\mathbf{P}$ \\
\hline \multirow{4}{*}{$\begin{array}{l}\text { Sleep quality } \\
\text { During the past month, how would you rate your sleep quality } \\
\text { overall? }\end{array}$} & Very good & 2 & 2.9 & 40 & 57.1 & \multirow{4}{*}{132.36} & \multirow{4}{*}{$<0.001 * *$} \\
\hline & Fairly good & 3 & 4.3 & 26 & 37.1 & & \\
\hline & Fairly bad & 38 & 54.3 & 2 & 2.9 & & \\
\hline & Very bad & 27 & 38.5 & 2 & 2.9 & & \\
\hline \multirow{4}{*}{$\begin{array}{l}\text { Sleep latency } \\
\text { How long has it taken you to fall asleep each night? }\end{array}$} & $<15$ minutes & 6 & 8.6 & 65 & 92.8 & \multirow{4}{*}{117.89} & \multirow{4}{*}{$<0.001 * *$} \\
\hline & 16-30 minutes & 44 & 62.8 & 2 & 2.9 & & \\
\hline & $31-60$ minutes & 17 & 24.3 & 1 & 1.4 & & \\
\hline & $>60$ minutes & 3 & 4.3 & 2 & 2.9 & & \\
\hline & Not during the past month & 4 & 5.7 & 37 & 52.8 & & \\
\hline During the past month, how often have you had trouble & Less than once/week & 2 & 2.9 & 28 & 40.0 & & O $001 * *$ \\
\hline sleeping because you... & Once or twice/week & 10 & 14.3 & 3 & 4.3 & 117.95 & $<0.001$ F. \\
\hline & Thrice or more/week & 54 & 77.1 & 2 & 2.9 & & \\
\hline & $>7$ hours & 23 & 32.8 & 3 & 4.3 & & \\
\hline Sleep duration & 6-7 hours & 42 & 60.0 & 2 & 2.9 & & \\
\hline How many hours of sleep do you get at night actually? & 5-6 hours & 2 & 2.9 & 40 & 57.1 & 132.36 & $<0.001^{* *}$ \\
\hline & $<5$ hours & 3 & 4.3 & 25 & 35.7 & & \\
\hline Sleep efficiency: & $>85 \%$ & 2 & 2.9 & 2 & 2.9 & & \\
\hline When have you usually gone to bed? When have you usually & $75-84 \%$ & 25 & 35.7 & 32 & 45.7 & & \\
\hline gotten up in the morning? & $65-74 \%$ & 22 & 31.4 & 34 & 48.5 & 21.85 & $<0.001^{* *}$ \\
\hline How many hours of actual sleep do you get at night? & $<65 \%$ & 21 & 30.0 & 2 & 2.9 & & \\
\hline Sleep disturbance & Not during the past month & 2 & 2.9 & 26 & 37.1 & & \\
\hline During the past month, how often have you had trouble sleeping & Less than once/week & 2 & 2.9 & 39 & 55.7 & 13236 & $\leq 0 \Omega 01 * *$ \\
\hline because you... & Once or twice/week & 16 & 22.8 & 3 & 4.3 & 132.36 & 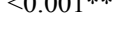 \\
\hline Wake up in the middle of the night or early morning & Thrice or more/week & 50 & 71.4 & 2 & 2.9 & & \\
\hline & Not during the past month & 4 & 5.7 & 18 & 25.7 & & \\
\hline Get un to use the bathroom & Less than once/week & 10 & 14.3 & 47 & 67.1 & 0333 & $\leq 0 \Omega 01 * *$ \\
\hline Get up to use the bathroom & Once or twice/week & 12 & 17.1 & 2 & 2.9 & 93.33 & 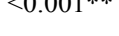 \\
\hline & Thrice or more/week & 44 & 62.9 & 3 & 4.3 & & \\
\hline & Not during the past month & 2 & 2.9 & 37 & 52.8 & & \\
\hline$C_{0} 1 \mathrm{~b}$ - & Less than once/week & 2 & 2.9 & 28 & 40.0 & 1205 & $0001 * *$ \\
\hline Can't breathe comfortably & Once or twice/week & 16 & 22.8 & 3 & 4.3 & 132.5 & $<0.001 * *$ \\
\hline & Thrice or more/week & 50 & 71.4 & 2 & 2.9 & & \\
\hline & Not during the past month & 2 & 2.9 & 14 & 20.0 & & \\
\hline & Less than once/ week & 3 & 4.3 & 50 & 71.4 & 11807 & $<0001 * *$ \\
\hline Cough or snore loudly & Once or twice/week & 18 & 25.7 & 4 & 5.7 & 118.97 & $<0.001$ * \\
\hline & Thrice or more/week & 47 & 67.1 & 2 & 2.9 & & \\
\hline & Not during the past month & 18 & 25.7 & 47 & 67.1 & & \\
\hline Eeol ton cold & Less than once/week & 1 & 1.4 & 18 & 25.7 & & \\
\hline Feel too cold & Once or twice/week & 22 & 31.4 & 2 & 2.9 & 87.05 & $<0.001 * *$ \\
\hline & Thrice or more/week & 29 & 41.5 & 3 & 4.3 & & \\
\hline & Not during the past month & 6 & 8.6 & 40 & 57.1 & & \\
\hline & Less than once/week & 2 & 2.9 & 25 & 35.7 & 11152 & $-0001 * *$ \\
\hline Feel too hot & Once or twice/week & 22 & 31.4 & 3 & 4.3 & 111.53 & $<0.001^{* *}$ \\
\hline & Thrice or more/week & 40 & 57.1 & 2 & 2.9 & & \\
\hline & Not during the past month & 10 & 14.3 & 52 & 74.3 & & \\
\hline Haye bad 20 a & Less than once/week & 24 & 34.3 & 15 & 21.4 & 6785 & $<0001 * *$ \\
\hline Have bad dreams & Once or twice/week & 14 & 20.0 & 1 & 1.4 & & $<0.001^{*}$ \\
\hline & Thrice or more/week & 22 & 31.4 & 2 & 2.9 & & \\
\hline & Not during the past month & 6 & 8.6 & 38 & 54.2 & & \\
\hline Науе nain & Less than once/week & 4 & 5.7 & 27 & 38.6 & 10501 & $\leq 0001 * *$ \\
\hline Have pain & Once or twice/week & 26 & 37.1 & 2 & 2.9 & 105.01 & \\
\hline & Thrice or more/week & 34 & 48.6 & 3 & 4.3 & & \\
\hline & Not during the past month & 46 & 65.7 & 53 & 75.7 & & \\
\hline Please describe, how often you have had trouble sleeping because & Less than once/week & 2 & 2.9 & 14 & 20.0 & 3809 & $<0001 * *$ \\
\hline of other reason $(\mathrm{s})$ : & Once or twice/week & 12 & 17.1 & 2 & 2.9 & 38.98 & $<0.001$ a \\
\hline & Thrice or more/week & 10 & 14.3 & 1 & 1.4 & & \\
\hline & Not during the past month & 16 & 22.8 & 53 & 75.7 & & \\
\hline Sleep with medications & Less than once/week & 2 & 2.9 & 12 & 17.1 & (2) & $\Omega_{0} \cap 01 *$ \\
\hline How often have you taken medicine to help you sleep? & Once or twice/week & 30 & 42.9 & 3 & 4.3 & 82.98 & $<0.001$ F. \\
\hline & Thrice or more/week & 22 & 31.4 & 2 & 2.9 & & \\
\hline & Not during the past month & 2 & 2.9 & 37 & 52.8 & & \\
\hline Day time dysfunction & Less than once/week & 3 & 4.3 & 27 & 38.6 & 14074 & $\leq 0 \Omega 01 * *$ \\
\hline How often have you had trouble staying awake while driving, & Once or twice/week & 24 & 34.3 & 3 & 4.3 & 140.74 & $<0.001^{* *}$ \\
\hline & Thrice or more/week & 41 & 58.5 & 3 & 4.3 & & \\
\hline & Not during the past month & 2 & 2.9 & 45 & 64.2 & & \\
\hline How much a problem has it been for you to keep up enthusiasm to & Less than once/week & 2 & 2.9 & 20 & 28.6 & 14001 & $=000$ \\
\hline get things done? & Once or twice/week & 31 & 44.2 & 3 & 4.3 & 140.01 & $<0.001^{*}$ \\
\hline & Thrice or more/week & 35 & 50.0 & 2 & 2.9 & & \\
\hline
\end{tabular}

**p-value $<0.001$ (HS) 
Table 3. Descriptive Statistics for Fatigue Severity Items Scores Pre and Post Palliative Care Intervention (n=70).

\begin{tabular}{|c|c|c|c|c|c|c|}
\hline \multirow{2}{*}{ Fatigue severity items } & \multicolumn{2}{|c|}{ Pre intervention } & \multicolumn{2}{|c|}{ Post intervention } & \multicolumn{2}{|c|}{ Chi square test } \\
\hline & No & $\%$ & No & $\%$ & $\mathrm{X}^{2}$ & P-value \\
\hline \multicolumn{7}{|c|}{$\begin{array}{l}\text { During the past week, I have found that: } \\
\text { My motivation is decreased when I am fatigued. }\end{array}$} \\
\hline Strongly disagree & 3 & 4.3 & 21 & 30.0 & \multirow{5}{*}{114.15} & \multirow{5}{*}{$<0.001 * *$} \\
\hline Disagree & 2 & 2.9 & 34 & 48.6 & & \\
\hline Neutral & 13 & 18.6 & 11 & 15.7 & & \\
\hline Agree & 26 & 37.1 & 2 & 2.9 & & \\
\hline Strongly agree & 26 & 37.1 & 2 & 2.9 & & \\
\hline \multicolumn{7}{|l|}{ Exercise brings fatigue for me. } \\
\hline Strongly disagree & 2 & 2.9 & 33 & 47.1 & \multirow{5}{*}{140} & \multirow{5}{*}{$<0.001 * *$} \\
\hline Disagree & 1 & 1.4 & 31 & 44.3 & & \\
\hline Neutral & 8 & 11.4 & 2 & 2.9 & & \\
\hline Agree & 27 & 38.6 & 3 & 4.3 & & \\
\hline Strongly agree & 32 & 45.7 & 1 & 1.4 & & \\
\hline \multicolumn{7}{|l|}{ I am easily fatigued. } \\
\hline Strongly disagree & 2 & 2.9 & 15 & 21.4 & \multirow{5}{*}{126.28} & \multirow{5}{*}{$<0.001^{* *}$} \\
\hline Disagree & 1 & 1.4 & 42 & 60.6 & & \\
\hline Neutral & 6 & 8.6 & 8 & 11.4 & & \\
\hline Agree & 24 & 34.3 & 3 & 4.3 & & \\
\hline Strongly agree & 37 & 52.9 & 2 & 2.9 & & \\
\hline \multicolumn{7}{|l|}{ Fatigue affects my physical function. } \\
\hline Strongly disagree & 2 & 2.9 & 13 & 18.6 & \multirow{5}{*}{110.13} & \multirow{5}{*}{$<0.001 * *$} \\
\hline Disagree & 1 & 1.4 & 38 & 54.3 & & \\
\hline Neutral & 15 & 21.4 & 15 & 21.4 & & \\
\hline Agree & 26 & 37.1 & 3 & 4.3 & & \\
\hline Strongly agree & 26 & 37.1 & 1 & 1.4 & & \\
\hline \multicolumn{7}{|l|}{ Fatigue causes recurrent problems for me. } \\
\hline Strongly disagree & 2 & 2.9 & 19 & 27.1 & \multirow{5}{*}{112} & \\
\hline Disagree & 1 & 1.4 & 34 & 48.6 & & \\
\hline Neutral & 13 & 18.6 & 14 & 20.0 & & $<0.001^{* *}$ \\
\hline Agree & 39 & 55.7 & 1 & 1.4 & & \\
\hline Strongly agree & 15 & 21.4 & 2 & 2.9 & & \\
\hline My fatigue prevents continued physical & & & & & & \\
\hline Strongly disagree & 2 & 2.9 & 15 & 21.4 & & \\
\hline Disagree & 3 & 4.3 & 34 & 48.6 & & \\
\hline Neutral & 9 & 12.9 & 18 & 25.7 & 114.28 & $<0.001^{* *}$ \\
\hline Agree & 38 & 54.3 & 2 & 2.9 & & \\
\hline Strongly agree & 18 & 25.7 & 1 & 1.4 & & \\
\hline Fatigue interferes with performing cert & & & & & & \\
\hline Strongly disagree & 2 & 2.9 & 11 & 15.7 & & \\
\hline Disagree & 3 & 4.3 & 40 & 57.1 & & \\
\hline Neutral & 22 & 31.4 & 14 & 20.0 & 101.6 & $<0.001^{* *}$ \\
\hline Agree & 22 & 31.4 & 2 & 2.9 & & \\
\hline Strongly agree & 21 & 30.0 & 3 & 4.3 & & \\
\hline Fatigue is among the most disabling syl & & & & & & \\
\hline Strongly disagree & 2 & 2.9 & 16 & 22.9 & & \\
\hline Disagree & 3 & 4.3 & 18 & 25.7 & & \\
\hline Neutral & 24 & 34.3 & 32 & 45.7 & 81.06 & $<0.001^{* *}$ \\
\hline Agree & 24 & 34.3 & 3 & 4.3 & & \\
\hline Strongly agree & 17 & 24.3 & 1 & 1.4 & & \\
\hline Fatigue interferes with my work, family & & & & & & \\
\hline Strongly disagree & 2 & 2.9 & 15 & 21.4 & & \\
\hline Disagree & 1 & 1.4 & 36 & 51.4 & & \\
\hline Neutral & 21 & 30.0 & 14 & 20.0 & 102.94 & $<0.001^{* *}$ \\
\hline Agree & 25 & 35.7 & 3 & 4.3 & & \\
\hline Strongly agree & 21 & 30.0 & 2 & 2.9 & & \\
\hline
\end{tabular}

**p-value $<0.001$ (HS). 


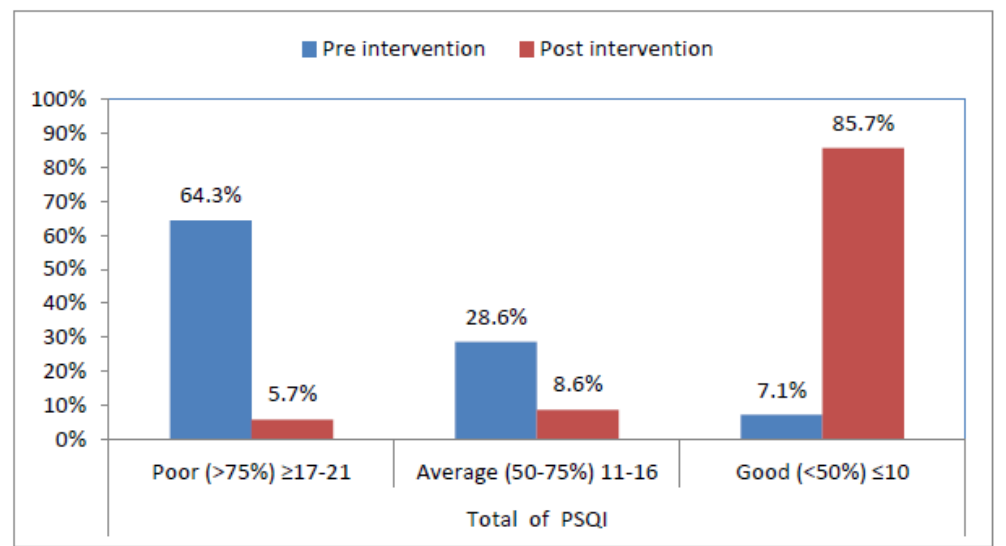

Figure 1. Total Scores of Sleep Quality for Clients' with Chronic Obstructive Pulmonary Disease Pre and Post Palliative Care Intervention $(* *$ p-value $<0.001 \mathrm{HS})$

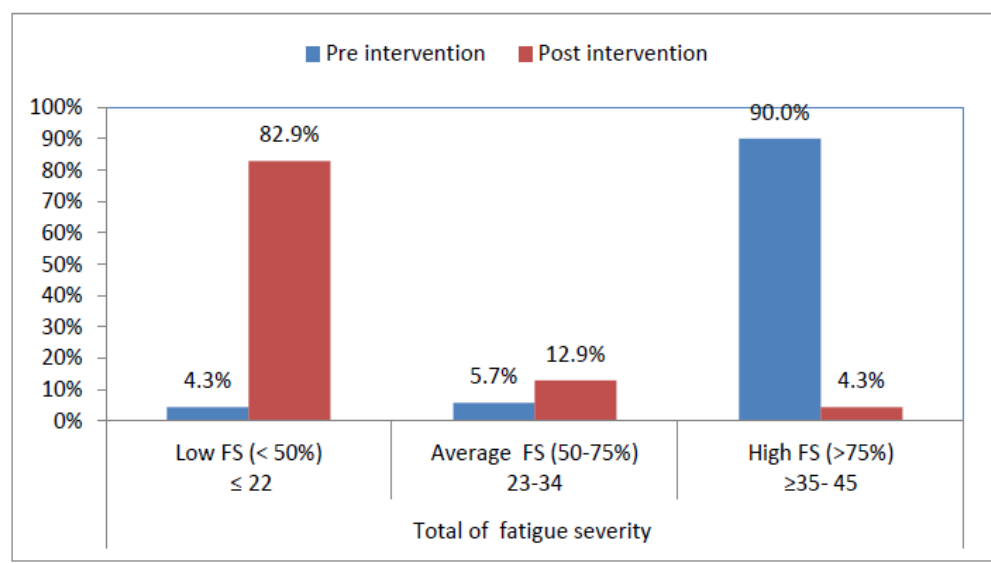

Figure 2. Total Scores of Fatigue Severity for Clients' with Chronic Obstructive Pulmonary Disease Pre and Post Palliative Care Intervention $(* *$ p-value $<0.001 \mathrm{HS})$

\section{In relation to $\mathrm{H} 1$ :}

Table 4. Comparison between Pre and Post Palliative Care Intervention for Clients with Chronic Obstructive Pulmonary Disease According to their Pittsburgh Sleep Quality and Total Fatigue Severity Mean Score $(n=70)$

\begin{tabular}{|l|c|c|c|c|}
\hline \multicolumn{1}{|c|}{ Items } & Pre intervention & Post intervention & \multicolumn{2}{c|}{ t-test } \\
\hline Pittsburgh sleep quality & \multicolumn{3}{|c|}{} & \multicolumn{2}{c|}{} \\
\hline Sleep quality & $1.83 \pm 1.12$ & $0.17 \pm 0.38$ & 11.76 & $<0.001^{* *}$ \\
\hline Sleep latency & $2.74 \pm 0.56$ & $0.69 \pm 0.47$ & 23.68 & $<0.001^{* *}$ \\
\hline Sleep duration & $2.40 \pm 0.55$ & $0.43 \pm 0.50$ & 22.24 & $<0.001^{* *}$ \\
\hline Habitual sleep efficiency & $1.94 \pm 0.83$ & $1.51 \pm 0.61$ & 3.48 & $<0.001^{* *}$ \\
\hline Sleep disturbance & $2.63 \pm 0.49$ & $1.03 \pm 0.29$ & 23.56 & $<0.001^{* *}$ \\
\hline Sleeping with medication & $2.63 \pm 0.49$ & $0.43 \pm 0.50$ & 26.42 & $<0.001^{* *}$ \\
\hline Day time dysfunction & $2.54 \pm 0.56$ & $0.51 \pm 0.50$ & 22.62 & $<0.001^{* *}$ \\
\hline Total PSQI & $16.71 \pm 2.81$ & $4.77 \pm 1.62$ & 30.79 & $<0.001^{* *}$ \\
\hline Total fatigue severity & $54.83 \pm 5.42$ & $17.80 \pm 4.68$ & 43.27 & $<0.001^{* *}$ \\
\hline
\end{tabular}

$* *$ p-value $<0.001$ (HS).

\section{According to H2:}

Table 5. Correlation between Total Fatigue Severity and Pittsburgh Sleep Quality (n=70).

\begin{tabular}{|l|c|c|}
\hline \multicolumn{1}{|c|}{ Pittsburgh sleep quality } & r & \multicolumn{2}{c|}{ Total fatigue severity } \\
\cline { 2 - 3 } & 0.722 & $<0.001^{* *}$ \\
\hline Sleep quality & 0.881 & $<0.001^{* *}$ \\
\hline Sleep latency & 0.864 & $<0.001^{* *}$ \\
\hline Sleep duration & 0.270 & $<0.001^{* *}$ \\
\hline Habitual sleep efficiency & 0.887 & $<0.001^{* *}$ \\
\hline Sleep disturbance & 0.910 & $<0.001^{* *}$ \\
\hline Sleeping with medication & 0.905 & $<0.001^{* *}$ \\
\hline Day time dysfunction & 0.930 & $<0.001^{* *}$ \\
\hline Total PSQI & & \\
\hline
\end{tabular}

r: Spearman's rank correlation coefficient

$* *$ p-value $<0.001$ (HS). 
Figure 2: Reveals that $90 \%$ of the clients had high fatigue severity pre intervention and improved and reduced to reach $4.3 \%$ post intervention with a highly statistically significant difference in comparison to pre intervention $(\mathrm{p}=<0.001)$.

Table 4: Shows highly statistically significant differences between pre and post palliative care intervention for clients' with chronic obstructive pulmonary disease in all items of Pittsburgh sleep quality and total fatigue severity $(\mathrm{p}=<0.001)$.

Table 5: Displays that there were highly statistically significant positive correlations between total fatigue severity and all components of Pittsburgh sleep quality index among chronic obstructive pulmonary disease clients $(\mathrm{p}=<0.001)$.

\section{Discussion}

Chronic obstructive pulmonary disease clients might experience fatigue that results from sleeplessness and decrease of the oxygen capacity [23]. Palliative care intervention increases the quality of life for these clients who are facing problems associated with life threatening disease, through the prevention and mitigate of suffering [24].

Concerning the demographic characteristics of the studied clients with COPD, the present study revealed that, for more than two thirds of the studied clients their age was between $>30-40$ years. This was due to avoid clients with co-morbid conditions that may affect the results and with age range 20-40 years. These results are in agreement with those of [25], in Egypt, about "effect of care protocol on the knowledge, practice and clinical outcomes of patients with chronic obstructive pulmonary disease", which mentioned that around two thirds of the studied sample were in age between 30-50 years. However, this finding was incongruent with study done by [26], about "preference for different relaxation techniques by COPD patients", which reported that, the mean age of Caucasian patients with COPD was 67 years.

In the present study finding, less than two thirds of studied clients were male, this finding may be due to the increased prevalence of smoking among this gender and the occupational exposure of them to polluted work environment more than females. This finding was supported by that of [27], in Egypt, about "quality of life in COPD patients", which stated that the majority of patients were male. However, this study result was in disagreement with that of [28], in North Shore-Long Island, about "Effect of pulmonary rehabilitation on fatigue Severity Scale in patients with lung disease", which reported that, more than half of the study subjects were female. Additionally, the [29], which mentioned that, females are more likely to have COPD than males.

In relation to educational level, the current study result showed that, an equal percentage of two fifths of clients were either primary, or university. This was due to inclusion criteria of this study to allow good understanding and application of the study intervention. These results were disagreement with those of [30], in the south of the Dutch, about "improving quality of life in depressed COPD patients", who found that more than one third of the studied subjects had low educational level.
As regards marital status, the results of the present study revealed that, nearly three quarters of the clients were married. This finding is supported by [31], in Brazil, about "influence that socio-demographic on quality of life in COPD patients on long-term home oxygen therapy", who mentioned that, more than half of the studied sample were married. This may reflect the load, as well as the higher levels of anxiety and stress experienced by the clients. These married individuals are a burden on their families because of the decrease expected roles in their life.

Regarding working type, this study finding showed that more than half of the studied sample were working as carpenters, bakers and shoe repair. So, this result conveys that these clients are more likely to have the disease because they were exposed to more pollutions that are risk factors for developing the disease. This result is in accordance with that of [32], in Egypt, about" effect of care protocol on the knowledge, practice and clinical outcomes of patients with chronic obstructive pulmonary disease", who reported that, less than three fifths of the studied subjects were exposed to irritating works.

The current study finding indicated that, there were highly statistically significant differences in all components of PSQI mean scores (Sleep quality, latency, duration, efficiency, disturbance, sleep with medications and day time dysfunction) between pre/post palliative care intervention application. This may be due to the continuous performance of the exercise intervention that affected positively on sleep quality. These findings were supported by those of [25], which revealed that there were highly statistically significant differences between mean scores of total sleeping pattern pre and post implementation of a care protocol for COPD patients.

Similarly, these results were similar to those of [33], in Italy, about "relaxation techniques for people with chronic obstructive pulmonary disease", which found that training sessions of relaxation techniques could decrease anxiety and produce benefits for some physiological parameters that have positive effect in quality of sleep in persons with COPD.

As regards fatigue severity items scores pre and post palliative care intervention, these study findings showed that there were highly statistically significant differences in all items of fatigue severity scores pre/post palliative care intervention. These findings were in agreement with those of the study done in Indonesia by [34], about "the effect of breathing exercises on the fatigue levels of patients with chronic obstructive pulmonary disease", who reported that training of respiratory exercises through face to face procedure while implementing treatment procedures may have significant effects on controlling fatigue and improving the symptoms. As well, [35], in Egypt, about "chronic obstructive pulmonary disease: Effect of nursing intervention protocol on clinical patients' outcomes post-weaning from mechanical ventilation", who explained that there was statistically significant change in the mean score of patients' fatigue post pulmonary rehabilitation program in both groups.

The results of the present study displayed that, there were highly statistically significant correlations between pre and post palliative care intervention for clients' with COPD in all items of Pittsburgh sleep quality index and 
total fatigue severity scale. In addition, the Pittsburgh sleep quality index was improved post palliative care intervention with highly statistically significant difference in comparison to pre intervention. This results was in the same line with that of a study done in East of Turkey, by [36], about "effect of progressive relaxation exercises on fatigue and sleep quality in patients with chronic obstructive lung disease", who mentioned that, progressive relaxation exercises have positive effects on fatigue severity and sleep quality in patients with COPD and found statistically significant difference in global sleep quality and patients' sleep quality improving in the group applying the progressive relaxation exercises.

This may be due to the application of the palliative care intervention and relaxation technique which became an integral part of the care of clients with COPD because of its benefits as reducing anxiety and stress, facilitating sleep, and mitigate fatigue. Additionally, this reflects the effectiveness of the palliative care intervention in mitigating the sleep inefficiency among COPD clients.

The current study result reveals that fatigue severity was improved post intervention with a highly statistically significant difference in comparison to pre intervention. This result was supported by [37], in Uppsala, Sweden, about "physical activity and fatigue in chronic obstructive pulmonary disease", who clarified that fatigue was higher in subjects with low physical activity among those with COPD. Recently, this result also was similar to that of [38], in Iran, about "the effect of progressive muscle relaxation on the management of fatigue and quality of sleep in patients with chronic obstructive pulmonary disease", who found that progressive muscle relaxation program reduces fatigue, but has no effect on some subscales of sleep quality as sleep disturbances, use of sleeping medications, and daytime dysfunction. This may be owing to the fact that exercises and relaxation positively improves pulmonary function and thus reflect on mitigating dyspnea, fatigue and also sleep inefficiency.

The above mentioned results proved the aim of the present study and justified its first hypothesis, which revealed that the palliative care intervention will mitigate fatigue severity and sleep inefficiency to COPD clients.

The findings of the current study displayed that there were highly statistically significant positive correlations between total fatigue severity score and all components of sleep quality among COPD clients. These results were similar to those of a study conducted in Iran, by [9], about "the relationship between sleep quality with fatigue severity and academic performance", which clarified that a significant relation between sleep quality and fatigue severity $(\mathrm{p} \leq 0.000)$.

The above mentioned results proved the second hypothesis of the present study, which revealed that, there will be a significant relation between fatigue severity and sleep quality to COPD clients.

\section{Conclusion}

The palliative care intervention had a positive effect on chronic obstructive pulmonary disease clients and helped them to mitigate sleep inefficiency, which led to reduce fatigue. As well, the results showed highly statistically significant positive correlations between fatigue severity and all components of sleep quality index among chronic obstructive pulmonary disease clients.

\section{Recommendations}

- An illustrated booklet containing information about benefits of gradual relaxation exercises should be available in all chest outpatient clinics.

- Training of the nurses on how to apply the palliative care intervention to chronic obstructive pulmonary disease clients to cope with their disease. Counseling for public about the importance of exercises for chronic obstructive pulmonary disease.

\section{References}

[1] Badway, M.Sh., Hamed, A.F., \& Yousef, F.M.A. (2016): Prevalence of chronic obstructive pulmonary disease (COPD) in Qena Governorate. Egyptian Journal of Chest Diseases and Tuberculosis 65: 29-34.

[2] World Health Organization (WHO). (2015): Chronic obstructive pulmonary disease (COPD). Retrieved from: http://www.who.int.

[3] Centers for Disease Control and Prevention. (2017). Chronic obstructive pulmonary disease (COPD). Available at: https://www.cdc.gov/copd/index.html. Accessed on: February 6, 2018.

[4] Said, A.F., Ewis, A.A., Omran, A.A., Magdy, M.E., \& Saleeb, M.F. (2015). Prevalence and predictors of chronic obstructive pulmonary disease among high-risk Egyptians. Egyptian Journal of Broncholog, 9 (1):27-33.

[5] Tashkin, D.P. (2015): Does marijuana pose risks for chronic airflow obstruction? Annals of the American Thoracic Society, 12(2), 235-6.

[6] Fabbri, L.M. (2016). Smoking not COPD, as the disease. N. Engl. J. Med., 374: 1885-1886.

[7] Lewko, A., Bidgood, P.L., Jewell, A., \& Garrod, R. (2014). Evaluation of multidimensional COPD-related subjective fatigue following a pulmonary rehabilitation program. Respiratory Medicine., 108(1): 95-102.

[8] Aydin, H.T. (2014): Chronic obstructive pulmonary disease and sleep quality. Chapter 6, COPD Clinical Perspectives. Licensee In Tech. Accessed at: 7/7/2018.

[9] Sajadi, A., Farsi, Z., \& Rajai, N. (2014). The relationship between sleep quality with fatigue severity and academic performance of nursing students. Nursing Practice Today; 1(4): 213-220.

[10] Soler, X., Diaz-Piedra, C., \& Ries, A. L. (2013): Pulmonary rehabilitation improves sleep quality in chronic lung disease. Journal of Chronic Obstructive Pulmonary Disease; 10(2): 156-63.

[11] Cancelliero-Gaiad, K.M., Ike, D., Pantoni, C.B., Borghi-Silva, A., \& Costa, D. (2014): Respiratory pattern of diaphragmatic breathing and Pilates breathing in COPD subjects. Brazilian Journal of Physical Therapy; 18(4):291-9.

[12] Campion, E.W., Kelley, A.S., \& Morrison, R.S. (2015): Palliative care for the seriously ill. New England Journal of Medicine; 373(8): 747-55

[13] Lobato, S., \& Alises, S. (2015): Palliative care in COPD patients: A challenge in modern medicine. Lung India, 32: 6.

[14] The American Lung Association. (2017): Lung health \& diseases, palliative care and COPD. Available at: https://www.lung.org/lung-health - and - diseases/lung - diseaselookup/copd/diagnosing - and -treating/palliative-care-andcopd.html. Date of access: March 13, 2018.

[15] Nursing Management. (2017): Chronic obstructive pulmonary disease. Chapter 27, respiratory problems, unit 6, pp. 680-684. Accessed date: 16/12/2017.

[16] Nurse Registry. (2018). The role of the nurse in palliative care What is Palliative Care? Available at: https://www.nurseregistry.com/blog/palliative-care-nursing/ accessed date: $1 / 9 / 2018$ 
[17] McCarthy, B. D., Casey, D., Devane, K., Murphy, E., Murphy, Y., \& Lacasse. (2015): Pulmonary rehabilitation for chronic obstructive pulmonary disease, Cochrane Library Syst. Rev. 2.

[18] Egypt Ministry of Health Conference. (2018): Three million Egyptians at risk of chronic obstructive pulmonary disease and early diagnosis lifeline for patients, Elmogaz Journal. Available at: http://www.elmogaz.com/node/454737. Accessed date: 29 March 2018.

[19] Liu, X.L., Tan, J.Y., Wang, T., Zhang, Q., Zhang, M., \& Yao, L.Q. (2014): Effectiveness of home based pulmonary rehabilitation for patients with chronic obstructive pulmonary disease: A metaanalysis of randomized controlled trials. Rehabilitation Nursing; 39(1):36-59.

[20] Chow, S.C., Shao, J., \& Wang, H. (2007): Sample size calculation in clinical research, Second edition. Chapman and Hall/CRC Biostatistics Series, pp. 389-405.

[21] Buysse, D.J., Reynolds, C.F., Monk, T.H., Berman, S.R., \& Kupfer, D.J. (1989): The Pittsburgh Sleep Quality Index (PSQI) A new instrument for psychiatric research and practice. Psychiatry Research; 28:193-213.

[22] Krupp, L.B., LaRocca, N.G., Muir-Nash, J., \& Steinberg, A.D (1989): The Fatigue Severity Scale. Application to patients and measures fatigue severity in a variety of medical and neurologic disorders Arch Neurol. Oct; 46(10):1121-3.

[23] Wong, C.J.D. Goodridge, D.D., Marciniuk, D., \& Rennie. (2010): Fatigue in patients with COPD participating in a pulmonary rehabilitation program. Int. J. Chron. Obstruct. Pulmon. Dis; 5: 319-326.

[24] Disler, R., Inglis, S.C., Currow, D.C., Davidson, P.M. (2012): Palliative and supportive care in chronic obstructive pulmonary disease: Research Priorities to Decrease Suffering. 1:301. 1: 6 .

[25] Maarouf, M. (2016): Effect of care protocol on the knowledge, practice and clinical outcomes of patients with chronic obstructive pulmonary disease, Doctorate thesis, Faculty on Nursing, Ain Shams University.

[26] Hyland, M.E., Halpin, D.M., Blake, S., Seamark, C., Pinnuck, M., Ward, D., \& Seamark, D. (2016): Preference for different relaxation techniques by COPD patients: Comparison between six techniques, International Journal of Chronic Obstructive Pulmonary Disease; 11(1): 2315-2319.

[27] Zamzam, M., Azab, N., El Wahsh, R., Ragab, A., \& Allam, E., (2012): Quality of life in COPD patients, The Egyptian Journal of Chest Diseases and Tuberculosis; 61: 281-289.

[28] Talwar, A., Sahni, S., Verma, S., Garcia, J., \& Kohn, N. (2014): Effect of pulmonary rehabilitation on Fatigue Severity Scale in patients with lung disease. Pneumonol. Alergol.; 82: $534-540$.
[29] Center for Disease Control and Prevention. (2013): Chronic obstructive pulmonary disease among adults-United States. Morbidity and Mortality Weekly Report; 61(46): 938- 43.

[30] Lamers, F., Jonkers, B. H., \& Chavannes, J. (2010). Improving quality of life in depressed COPD patients: Effectiveness of a minimal psychological intervention. COPD: Journal of Chronic Obstructive Pulmonary Disease; 7: 315-322 ISSN: 1541-2555. Accessed at: January 2019.

[31] Cedano, S., Belasco, A.G., Traldi, F., Malchado, M.C., \& Bettencourt, A.R. (2012): Influence that sociodemographic on quality of life in COPD patients on long-term home oxygen therapy. National Center for Biotechnology Information, U.S National Library of Medicine; 38(3): 331-8. Accessed at: January 2019.

[32] Mohamed, D., Ahmed, S.S., Mohamed, A.H., \& Abdel Rahman, A.A. (2017): Effect of care protocol on the knowledge, practice and clinical outcomes of patients with chronic obstructive pulmonary disease. Journal of Nursing Education and Practice; 7:2 Accessed date: 21/1/2019

[33] Volpato, E., Banfi, P., Rogers, S. \& Pagnini, F., (2015): Research article relaxation techniques for people with chronic obstructive pulmonary disease: A systematic review and a meta-analysis article. ID 628365, 22 pages. Accessed at: December 2018.

[34] Zakerimoghadam, M., Tavasoli, K., Nejad, A., \& Khoshkesht, S. (2011). The effect of breathing exercises on the fatigue levels of patients with chronic obstructive pulmonary disease. Acta Med Indones-Indones J Intern Med; 43 (1): 29-32.

[35] Salem, G., Mahdy, N., \& Abdelatief, D. (2017): Chronic obstructive pulmonary disease: Effect of nursing intervention protocol on clinical patients' outcomes post-weaning from mechanical ventilation. The 9th International Scientific Nursing Conference, Helwan University.

[36] Sahin, Z. A., \& Dayapoglu, N. (2015). Effect of progressive relaxation exercises on fatigue and sleep quality in patients with chronic obstructive lung disease (COPD). Complementary Therapies in Clinical Practice; 21: 277-281. Journal homepage: www.elsevier.com/locate/ctcp. Accessed at: December 2018.

[37] Andersson, M., Stridsman, C., Ronmark, E., Lindberg, A, \& Emtner, M. (2015): Physical activity and fatigue in chronic obstructive pulmonary disease. A population based study. 0954-6111. Elsevier Ltd. Available online at: www.sciencedirect.com. Accessed date: 22 May 2015.

[38] Chegeni, P.S., Gholami, M., Azargoon, A., Pour, A.H., Birjandi, M., \& Norollahi, H. (2018): The effect of progressive muscle relaxation on the management of fatigue and quality of sleep in patients with chronic obstructive pulmonary disease: A randomized controlled clinical trial. 1744-3881. Complementary Therapies in Clinical Practice; 31: 64-70. 